\title{
Worst Case Imputation Technique
}

National Cancer Institute

\section{Source}

National Cancer Institute. Worst Case Imputation Technique. NCI Thesaurus. Code C81203.

The substitution of missing data with the value that represents the worst possible outcome. 